\title{
Germanica
}

\section{Der Marquis von Keith de Frank Wedekind et Die Ehe des Herrn Mississippi de Friedrich Dürrenmatt : réappropriation subjective et déplacement de sens}

Frank Wedekinds Der Marquis von Keith und Friedrich Dürrenmatts Die Ehe des Herrn Mississippi : Einwirkung und Umfunktionierung.

Jacqueline Bel

\section{(2) OpenEdition}

\section{Journals}

\section{Édition électronique}

URL : http://journals.openedition.org/germanica/2070

DOI : 10.4000/germanica.2070

ISSN : 2107-0784

Éditeur

Université de Lille

\section{Édition imprimée}

Date de publication : 31 décembre 2002

Pagination : 71-83

ISBN : 9782913857100

ISSN : 0984-2632

\section{Référence électronique}

Jacqueline Bel, « Der Marquis von Keith de Frank Wedekind et Die Ehe des Herrn Mississippi de Friedrich Dürrenmatt : réappropriation subjective et déplacement de sens », Germanica [En ligne], 31 | 2002, mis en ligne le 19 mars 2013, consulté le 06 octobre 2020. URL : http://journals.openedition.org/ germanica/2070 ; DOI : https://doi.org/10.4000/germanica.2070

Ce document a été généré automatiquement le 6 octobre 2020.

(c) Tous droits réservés 


\section{Der Marquis von Keith de Frank Wedekind et Die Ehe des Herrn Mississippi de Friedrich Dürrenmatt : réappropriation subjective et déplacement de sens}

Frank Wedekinds Der Marquis von Keith und Friedrich Dürrenmatts Die Ehe des Herrn Mississippi : Einwirkung und Umfunktionierung.

Jacqueline Bel

C'est le 26 mars 1952 qu'une œuvre de Friedrich Dürrenmatt fut jouée pour la première fois en Allemagne. Il s'agissait de Die Ehe des Herrn Mississippi, une comédie dont le dramaturge avait commencé l'écriture en 1949 et qui avait déjà subi plusieurs remaniements avant la première représentation aux «Münchner Kammerspiele». Le texte de la pièce est publié la même année à Zurich, accompagné de la mention «Une comédie en deux parties ", alors qu'à l'origine Die Ehe des Herrn Mississippi se subdivisait en cinq actes. L'édition de 1952 reprend - au dire de l'auteur - « en gros »le texte joué à Munich. Quelques mois plus tard Tilly Wedekind dépose une plainte pour plagiat auprès du «Schutzverband deutscher Schriftsteller ». La veuve de Frank Wedekind argue avoir relevé des ressemblances frappantes entre le premier acte de Die Ehe des Herrn Mississippi et celui de Schloß Wetterstein ${ }^{1}$. Elle reproche en outre à Dürrenmatt d'avoir également plagié Erdgeist, Die Büchse der Pandora, Hidalla et Franziska. Dürrenmatt répond aux accusations de Tilly Wedekind dans Die Tat du 9 août 1952. L'article intitulé «Bekenntnisse eines Plagiators » déplore que Tilly Wedekind n'ait pas vu que la seule œuvre à avoir véritablement influencé l'écriture de Die Ehe des Herrn Mississippi était Der Marquis von Keith. C'est en effet à la lecture de cette pièce que Dürrenmatt a perçu la possibilité d'utiliser les personnages d'une manière dialectique et de leur faire incarner une idée ${ }^{2}$ - deux éléments spécifiques de sa conception de l'œuvre théâtrale. Alors que Tilly Wedekind s'applique à dénoncer les emprunts de détails opérés aux œuvres de son 
mari, l'auteur de Die Ehe des Herrn Mississippi dit avoir trouvé chez son confrère allemand quelque chose de "plus important " que des éléments ponctuels, à savoir la nécessité de recourir à la comédie ${ }^{3}$ pour rendre compte de son temps - une comédie d'un genre particulier, peuplée de cadavres. Trois ans plus tard, Dürrenmatt exposera ses théories dans les Theaterprobleme. Il estime que le persiflage est la seule façon de déranger « les tyrans de cette planète ».

\section{Réécriture des autres ou réécriture de soi ?}

2 Dürrenmatt était coutumier de la réécriture. Il a réécrit quatre fois Achterloo, sa dernière pièce - dont les personnages, les thèmes et les situations rappellent ceux de Die Ehe des Herrn Mississippi -, pour ne citer qu'un exemple. Peut-être en aurait-il rédigé d'autres versions si la mort n'avait pas mis un terme aux inventions de cet expérimentateur. Il lui arrivait non moins souvent de se plagier lui-même. Dans «Bekenntnisse eines Plagiators », il explique que Die Ehe des Herrn Mississippi poursuit le développement d'une idée incarnée par l'empereur et sa femme Julia dans Romulus der $G r o ß e^{4}$, comédie écrite en 1948 - non sans évoquer à cette occasion que dans Schloß Wetterstein Wedekind avait lui-même repris une situation qu'il avait trouvée dans Richard III de Shakespeare. L'écrivain, dramaturge, essayiste et peintre qu'est Dürrenmatt ne minimise pas l'influence de la littérature sur la production littéraire en général et la sienne en particulier, rejoignant ainsi Giraudoux qui considérait que «le plagiat est la base de toutes les littératures, excepté de la première, qui d'ailleurs est inconnue ». La lecture d'autres auteurs engage Dürrenmatt à retravailler des thèmes, des situations et des personnages qu'il incorpore selon ses propres critères et conceptions à ses œuvres, en les développant, les transformant ou les adaptant à l'esprit du temps, pour se démarquer de ses prédécesseurs. Dürrenmatt revendique ainsi le droit au soi-disant plagiat - en réalité une mise en question ou un élargissement pluridimensionnel de l'original. Les tenants sont similaires mais les aboutissants innovent. Die Ehe des Herrn Mississippi illustre justement la théorie de la réécriture de l'auteur, de la réécriture des autres mais aussi de soi. Dürrenmatt fait précéder le scénario tiré de la pièce Die Ehe des Herrn Mississippi en 1961 de la mention: « Ähnlichkeiten mit einem gleichnamigen Theaterstück eines gleichnamigen Autors sind rein zufällig $»^{5}$. Autrement dit, les similitudes sont volontaires. Le dramaturge suisse ne laisse rien au hasard dans ses écrits. C'est pourquoi il publie une deuxième version officielle de sa comédie en 1957, après la représentation de la pièce à Zurich. Or il retravaillera la pièce en 1960 à l'occasion d'une mise en scène au théâtre de la Bruyère à Paris, ce qui justifiera la parution d'une troisième version en 1964. Comme Die Ehe des Herrn Mississippi avait été portée à l'écran en 1961, Dürrenmatt republie le scénario en même temps que la troisième version revue et corrigée de la pièce en 1966. Viennent ensuite la quatrième et la cinquième version en 1972 et 1980 . L'auteur considère la dernière version, celle de 1980 , comme "une sorte de synthèse ${ }^{6}$, le résultat d'une évolution personnelle et de ses expériences. En ceci, il a suivi l'exemple de Wedekind qui a réécrit plusieurs fois Der Marquis von Keith et considérait cette pièce comme son œuvre la plus accomplie - dans la troisième et dernière version, les indications scéniques sont plus soignées que lors de la première édition et le texte épuré. Cependant, alors que le processus d'élaboration du texte est similaire chez les deux auteurs, la visée de Dürrenmatt diverge de celle de Wedekind. Il avait acquis la certitude que les hommes ne changent jamais bien que le monde soit en constante 
mutation - c'est l'idée centrale de Die Ehe des Herrn Mississippi. Ils continuent à être soumis à l'absurdité du hasard, à la contradiction et au paradoxe. Au regard de ces circonstances, la réécriture permanente, qui suit d'autres lois que celles de la logique, semble la seule adéquate. Dürrenmatt révise, corrige, adapte et actualise au fil des versions successives. Peter André Bloch décrit l'«art " auquel s'adonne Dürrenmatt comme un " anti-art " parce que ses techniques de composition théâtrale dérogent aux règles en vigueur. "Pour cela ", dit-il, «il a développé une dramaturgie paradoxale, énigmatique, qui cherche une expression pour l'inexprimable, des images pour l'inimaginable, des formules pour l'impensable $»^{7}$ - une dramaturgie qu'il a conçue par le biais d'une confrontation avec des auteurs tels que Frank Wedekind ou Bertolt Brecht.

\section{Die Ehe des Herrn Mississippi : un plagiat de Der Marquis von Keith?}

3 Les œuvres de Friedrich Dürrenmatt se nourrissent du réemploi de personnages, de noms, de situations, de lieux, de textes, de mythes, d'idéologies, de formes et de courants littéraires - Achterloo en est l'exemple par excellence - au point d'en devenir un mode de composition et d'écriture propre. Cette technique incite certains exégètes à réduire ce type de dramaturgie à un prétendu plagiat. L'article de Gerwin Marahrens, Frank Wedekinds Der Marquis von Keith und Friedrich Dürrenmatts Die Ehe des Herrn Mississippi ${ }^{8}$ rappelle - à l'instar d'autres exégètes d'ailleurs ${ }^{9}$ - l'influence exercée par Wedekind sur Dürrenmatt, notamment dans le cas de Die Ehe des Herrn Mississippi. Marahrens a effectué une analyse approfondie des parallélismes entre les deux pièces pour établir la filiation entre les deux œuvres :

Diese Arbeit möchte die Einwirkung von Wedekinds Marquis von Keith auf Dürrenmatts Die Ehe des Herrn Mississippi in allen Einzelheiten gründlich und erschöpfend untersuchen, vor allem in der Personengestaltung, der Metaphorik, der intellektuellen Artikulation, der Konzeption der Frauengestalten, des Bürgertums und der Gesellschaft und der Darstellung des Lebens als Theater und Jahrmarkt, und auf Gemeinsamkeiten und Verschiedenheiten der Gesellschaftsund Ideologiekritik hinweisen.

4 Les résultats de ses travaux confirment que Dürrenmatt connaissait bien Der Marquis von Keith et que des thèmes ou motifs de cette pièce apparaissent également dans Die Ehe des Herrn Mississippi. Dürrenmatt n'a pourtant pas pillé Wedekind, comme l'argumentation le sous-entend parfois. La comédie de Dürrenmatt n'est ni de la contrefaçon ni de la copie, encore moins de l'imitation. Elle frappe par la sobriété et la clarté de l'arrière-plan et du contexte, le choix de types représentatifs, la valeur d'universalité, la "globalisation » des événements et des personnages. À supposer que Dürrenmatt se soit appuyé sur Der Marquis von Keith pour élaborer Die Ehe des Herrn Mississippi, il aurait clarifié les situations, créé des personnages incarnant une idée et introduit ses thèmes de prédilection que sont la justice et la vérité, la religion et la politique, l'amour et l'argent. L'intention de Dürrenmatt se différencie radicalement de celle de Wedekind dans Schloß Wetterstein ou Der Marquis von Keith. Alors que Wedekind démasque l'hypocrisie de la morale bourgeoise dans une satire de la société munichoise du tournant du siècle, le théâtre de Dürrenmatt crée des mondes qui jouxtent le monde réel. Chez lui, l'idéologie est au centre des préoccupations de personnages qui aspirent à la réalisation d'un idéal et à la transformation du monde tandis que les personnages 
de Wedekind mènent un combat singulier pour accéder à un bonheur personnel lié à la reconnaissance sociale et à la sécurité matérielle. Wedekind conclut sur la difficulté de l'homme à maîtriser son existence - à la fin de la pièce, le marquis de Keith déclare : «Das Leben ist eine Rutschbahn... $»^{10}$ alors qu'une erreur d'appréciation lui avait fait croire à la possibilité de s'en rendre définitivement maitre : « Seit jenem Tage fühle ich mich erst wirklich als den Herrn meines Lebens ${ }^{11}$ - et Dürrenmatt sur l'impossibilité d'atteindre un objectif dicté par une idéologie: "So fielen wir, Henker und Opfer zugleich, durch unsere eigenen Werke $»^{12}$. Le dénouement comporte des " similitudes » puisque Casimir dans Der Marquis von Keith et Diego dans Die Ehe des Herrn Mississippi tirent profit des échecs de Keith chez Wedekind, de Mississippi et Saint-Claude chez Dürrenmatt, et que Übelohe d'une part, Keith et Ernst Scholz de l'autre - trois héritiers de Don Quichotte ${ }^{13}$ - ont le sentiment de se battre contre des moulins à vent. Celles-ci n'ont pourtant que valeur d'amorce pour le théâtre expérimental du dramaturge suisse. La comparaison des deux pièces permet en revanche de suivre le cheminement de la pensée de Dürrenmatt durant l'écriture et d'entrevoir les procédés dont il use. L'étude de Marahrens corrobore ainsi l'idée que Die Ehe des Herrn Mississippi n'est pas un calque de Der Marquis von Keith, mais bien «ein Experiment ${ }^{14}$, comme l'affirme Dürrenmatt. Gerwin Marahrens déduit pourtant des juxtapositions effectuées que :

\begin{abstract}
Vergleicht man abschließend den Marquis von Keith Wedekinds mit Dürrenmatts Die Ehe des Herrn Mississippi, so muß man zugeben, daß Wedekinds Schauspiel in einem stärkeren Ausmaß durchdacht und durchkonstruiert ist, mit äußerst zahlreichen, geradezu symmetrischen Entsprechungen, Verweisungen und wiederholten Spiegelungen arbeitet und ein bis in die letzten Einzelheiten durchgestaltetes Musterbeispiel für abstrahierende Modellhaftigkeit ist. Dürrenmatt übernimmt Wedekinds dramaturgische Methode, aber seine Praxis wird simpler und gröber, und zwar notwendigerweise, weil das ganze Leben und die Welt brutaler geworden sind und es nun um die Existenz der Menschheit und der Welt geht ${ }^{15}$.
\end{abstract}

Bien que l'article de Marahrens ne dénie pas à Dürrenmatt le caractère expérimental de Die Ehe des Herrn Mississippi, il juge que le dramaturge s'est simplifié la tâche en empruntant quantité de détails à Wedekind alors qu'il aurait pu et dû s'en remettre à son inspiration. En réalité, ces derniers ont joué le rôle de catalyseur chez Dürrenmatt qui applique dans Die Ehe des Herrn Mississippi une dramaturgie fondée sur la pensée ${ }^{16}$ dans laquelle réside toute la dignité de l'homme, selon Pascal. Cette dimension, étrangère à l'œuvre de Wedekind, est caractéristique du théâtre de Dürrenmatt et explique que Die Ehe des Herrn Mississippi et Der Marquis von Keith obéissent à des perspectives que leurs auteurs ont conçues autres. Cette constatation n'amoindrit ni n'augmente leurs mérites, mais souligne la divergence de leur conception du théâtre et, ce faisant, de leur stratégie - qui résulte elle-même de leur dessein.

\title{
Die Ehe des Herrn Mississippi : un champ d'expérimentation
}

6 À la différence de Wedekind pour lequel tables, chaises et portes servent de cadre à l'action, Dürrenmatt attribue le rôle principal aux décors scéniques et aux coulisses ${ }^{17}$, à une petite table ronde de style Biedermeier et à la pièce dans laquelle se trouve cette table que l'auteur qualifie de "personnage principal $»^{18}$. L'expérimentation se déroule dans un lieu ouvert et clos à la fois, en raison des nombreuses portes et fenêtres. Les meubles de style et la décoration intérieure font référence à divers continents et 
époques. Le lieu, le temps et l'action acquièrent une signification inhabituelle. Ils ont valeur symbolique d'universalité, d'intemporalité et d'exemplarité. Dürrenmatt procède à la fois en praticien et en théoricien. Selon lui, le théâtre se prête à l'exposition de théories et d'une conception du monde. Petit à petit, la pièce se délite ${ }^{19}$ sous l'action de l'échec des idéologues qui croient à la puissance de leurs idées au mépris de la réalité. L'auteur apporte la preuve "scientifique" de l'impossibilité de révolutionner le monde ou de le rendre meilleur. Les hommes se détruisent eux-mêmes - les principaux protagonistes sont empoisonnés ou abattus - dans leur lutte pour imposer des idéologies, mais ils ne peuvent détruire complètement le monde : la petite table ronde de style Biedermeier est le seul meuble indestructible qui résiste à toutes les épreuves. Le pragmatisme de la "Realpolitik" a le dernier mot. Le lieu et l'action ne constituent plus des unités distinctes, ils se fondent en un ensemble cohérent dont la forme fait également partie. La pièce de théâtre mêle action, commentaires de l'action en direction du public ${ }^{20}$ et indications scéniques données par les personnages ${ }^{21}$. Les retours en arrière et les projections dans l'avenir interrompent la linéarité et la chronologie du drame en combinant présent, passé et futur. Ainsi, les morts se relèvent et continuent à jouer. Ce procédé traduit aussi bien les tensions et la tendance au chaos d'un monde devenu problématique et invivable que l'invariabilité des lois énoncées par le texte. Dürrenmatt confère un sens nouveau aux éléments du drame bourgeois et moral de Wedekind. Il n'en va pas autrement dans l'exemple qui suit. Des piqueassiettes et des escrocs prennent place autour de la table dans le drame de Wedekind. Le marquis de Keith est utilisé par des parasites qui le dépossèdent. Dans Die Ehe des Herrn Mississippi, on prend apparemment le café entre gens de bonne compagnie. Mais on met du poison dans ce café, si bien que cette boisson et le jeu des tasses ${ }^{22}$ revêtent une importance fondamentale. Le rituel du café est à l'origine du mariage de Mississippi et de tout ce qui en découle. Chez Dürrenmatt, le fait que les personnages boivent le café crée une situation dramatique alors que chez Wedekind la visite des personnages à l'heure du petit-déjeuner relève de la satire sociale. Dürrenmatt fait dire à Übelohe dans son monologue sur fond de rideau fermé que l'auteur l'a «jeté dans le creuset de sa comédie $»^{23}$ - manifestement pour étudier les conséquences de sa mise en présence avec des situations particulières et d'autres créatures. L'auteur recompose le monde sur scène et synthétise les systèmes, les doctrines et les idéologies qui animent le monde réel dans le but d'analyser leur mode de fonctionnement et leurs effets sur la condition humaine.

\section{Une dramaturgie fondée sur la dialectique}

7 Le Marquis de Keith - en réalité roturier - et Ernst Scholz - en vérité Graf von Trautenau - figurent le matérialisme ${ }^{24}$ et la morale ${ }^{25}$ et prétendent respectivement à la respectabilité et au plaisir. Dürrenmatt a été conquis par cette méthode d'utilisation de la thèse et de l'antithèse qu'il a reprise dans la conception de ses personnages puisque Florestan Mississippi, Paule de son vrai nom, et Saint-Claude, qui se prénomme Louis, s'évertuent à répandre la loi de Moïse (la moralisation de la société par l'application d'une justice implacable ${ }^{26}$ et le communisme, deux convictions contraires. Ils sont opposés à Anastasia et au ministre Diego ${ }^{27}$, homme politique opportuniste et réaliste qui devra son accession au pouvoir à la faillite des idéaux de justice, d'égalité sociale, et de l'idéal de vérité et d'amour que prêche le comte Bodo von Übelohe-Zabernsee. Le drame de Dürrenmatt transcrit les aspirations de l'homme de son temps et met sa 
désorientation intellectuelle en évidence. Il transpose les questions d'ordre psychologique et individuel abordées par Wedekind dans le domaine philosophique et métaphysique, et leur attribue le caractère d'exemplarité.

Trois utopistes et idéologues se sont promis de changer et de sauver le monde ${ }^{28}$ en perdition par la loi de Moïse, le communisme et la religion chrétienne, trois religions, trois modes de salut : la loi, la politique et la foi. Ils échouent dans leur face à face avec Anastasia (Frau Welt) ${ }^{29}$, personnification du monde actuel, amoral, inconscient et dépourvu de scrupules, un monde qui résulte d'une réaction à ces trois modèles et qui périra lui aussi malgré sa vitalité. L'erreur des idéologies est de se considérer comme des dogmes dont la vérité n'est plus à prouver, de se transformer en culte et de susciter la foi et l'espoir bien qu'elles n'aient pas pour but de protéger l'homme. Elles deviennent dangereuses quand elles échouent, puisqu'elles mènent à l'anarchie et au chaos. Dans Der Marquis von Keith, les deux amis d'enfance Keith et Scholz font état de convictions personnelles opposées. Hermann Casimir coquette avec l'anarchie sans que les principes qu'il énonce entrent véritablement en contradiction ouverte avec ceux des deux autres personnages parce qu'il est adolescent et que ses certitudes sont susceptibles d'évoluer. Wedekind s'intéresse surtout aux passions et aux pulsions qui animent les hommes. Au dire de Dürrenmatt, il écrit encore de manière classique. Depuis, le théâtre a changé. La dramaturgie de l'auteur est inductive dans un premier temps, déductive ensuite. Tout en demeurant un art, son théâtre informe, documente, enseigne, émet des thèses et transmet des conclusions susceptibles de généralisation ${ }^{30}$. De même que l'écriture suppose une réflexion préalable chez le dramaturge, une représentation théâtrale doit déclencher un processus de réflexion chez le spectateur.

Die Ehe des Herrn Mississippi remplit le contrat établi par Dürrenmatt dans un discours intitulé "Vom Schreiben". Cette pièce de théâtre renseigne sur la nature, le mode de fonctionnement et la menace que font planer des idéologies incapables de dialoguer. Cette absence de communication est un des pires dangers de notre époque. Epris des idéologies censées donner un sens à leur vie, les idéologues n'essaient pas de se comprendre ou de se persuader mais de s'éliminer mutuellement ${ }^{31}$. Le fanatisme idéologique les déconnecte de principes fondamentaux tels que le respect de la loi ou la morale pour devenir une contradiction en soi destructrice, comme l'illustrent les exemples de Mississippi et de Saint-Claude. Les idéologies prospèrent depuis la disparition de la religion, des croyances et des valeurs éternelles; elles sont propres à la situation spirituelle et renaissent inlassablement de leurs cendres parce que l'homme a besoin de repères. Saint-Claude et Mississippi se relèvent après avoir été assassinés pour signifier l'impossibilité d'éradiquer les idéologies. Car les hommes s'abandonnent à Anastasia (le monde) et retournent à elle (à lui) même si elle est à l'origine de leur échec, étant donné que tous attendent quelque chose d'elle (de lui): la moralité, l'argent ou le pouvoir. Anastasia (le monde) est séduisante et amorale. On ne peut ni la changer ni la sauver. La série d'empoisonnements qui émaille la pièce de Dürrenmatt est symptomatique. Les idéologues intérieurement rongés par la haine tentent d'anéantir leur concurrent avec perfidie au lieu de se mesurer à lui par l'argumentation. Die Ehe des Herrn Mississippi expose en images la perversion de l'idée en idéologie et ses conséquences: l'absence de liberté de l'homme incapable de se libérer d'un carcan de compromissions sans le secours de la réflexion. Dürrenmatt se sert de fables pour réaliser l'objectif décrit dans "Vom Schreiben", à son avis le meilleur moyen d'exprimer les périls de son temps. 
Übelohe est le seul à garder foi en l'amour dans un monde en déliquescence. Ce Don Quichotte ${ }^{32}$ poursuit lui aussi infatigablement son combat, mais avec les armes de l'esprit. Les Don Quichotte du plaisir (le Marquis de Keith) et de la morale (Ernst Scholz) de Wedekind sont des personnages comiques qui croient à ce qu'il sont, d'où le tragique de leur situation. Le Don Quichotte de Dürrenmatt pratique l'ironie ${ }^{33}$. Il connaît le ridicule de ses actions et joue son rôle en conséquence. Übelohe est ainsi le seul personnage de Die Ehe des Herrn Mississippi à jouir de sa liberté et de la dignité humaine que confèrent la pensée et la dérision.

\section{La vie est un labyrinthe et le théâtre de Dürrenmatt son reflet}

11 Dans Der Marquis von Keith, Ernst Scholz souffre d'un mal existentiel tandis que les malheurs de Keith sont imputables à son caractère. Wedekind tente d'expliquer pour quelles raisons les personnages parviennent ou non au bonheur, quelles voies ils empruntent et en quoi réside la tragédie de leur existence. Die Ehe des Herrn Mississippi pose une question: est-il possible de changer le monde et les hommes? Le questionnement passe du particulier au général. Der Marquis von Keith s'interroge sur le monde et les hommes, Die Ehe des Herrn Mississippi en revanche crée un monde et le regarde fonctionner. Dans le théâtre de Dürrenmatt, l'homme veut fuir une vie qui l'agresse sans pouvoir lui échapper : Mississippi, Saint-Claude et Übelohe sont rattrapés par leur destin. C'est le drame de la condition humaine. Le monde est peuplé d'hommes qui souffrent et errent dans un labyrinthe, trompés par les puissants ou ceux qui détiennent un semblant de pouvoir. Malgré tout, l'homme doit supporter son temps au risque d'être condamné à l'implosion parce qu'il s'est compromis, qu'il a perdu sa liberté et sa dignité. La fuite dans la folie (Mississippi est interné, Ernst Scholz se rend de son propre chef dans un hôpital psychiatrique) ou l'acceptation de la mort sont les seules possibilités de les retrouver aussi bien chez Wedekind que chez Dürrenmatt. Mais les personnages du théâtre de Dürrenmatt sont des doubles du Minotaure emprisonné dans le labyrinthe - symbole de l'homme et du monde dans lequel il vit. L'homme ne cesse d'aspirer à la réalisation de soi, conscient que l'échec le guette et que le salut dans l'au-delà ou le bonheur terrestre promis par le marxisme sont des chimères. Chez Wedekind aussi, chacun des personnages aspire à l'épanouissement mais en saisissant toutes les occasions qui s'offrent à lui, alors que chez Dürrenmatt c'est le hasard qui règne car il s'est substitué à la grâce divine. C'est pourquoi l'auteur lui accorde une place de choix dans sa dramaturgie. Dürrenmatt ne croit plus en rien, il doute de tout, et sa dramaturgie se fonde sur cette dialectique. D'un autre côté, le hasard empêche sa dramaturgie de dégénérer en pure dialectique et de devenir ellemême idéologie et donc mécanisme. Le message que transmet Die Ehe des Herrn Mississippi est qu'il n'y a pas de solution idéale ni durable. Le doute demeure la seule croyance possible - ce que n'admettent ni Mississippi, pourfendeur du mal, ni SaintClaude, convaincu de la supériorité du communisme.

Dürrenmatt espère rendre le monde intelligible aux hommes par son théatre. Car la réalité du monde, dominé par des convictions défiant la raison, est effrayante. Un rationalisme cynique prétend vouloir délivrer l'homme d'un esclavage dont il est luimême responsable, le met en réalité à sa disposition et l'utilise pour réaliser sa volonté de puissance. Le théatre de Dürrenmatt part toujours d'une idée que l'auteur concrétise 
à l'aide d'images parce que les mots seuls sont impuissants à exprimer cette réalité. L'écriture est pour Dürrenmatt un moyen de se libérer d'un poids et une mise en garde. Il n'est pas moraliste comme Wedekind. Il communique son savoir, sa vision du monde. Il ne donne pas la solution pour sortir du labyrinthe. La comédie Die Ehe des Herrn Mississippi démontre de manière implacable le pire des tournants que peut prendre le monde et la vie des hommes. Les personnages n'éveillent pas la compassion malgré le sort qui est le leur. Bien que le monde que présente Die Ehe des Herrn Mississippi n'engage pas à l'espoir, Dürrenmatt attend des spectateurs qu'ils prennent la décision de résister à ce monde-là, de faire preuve de courage comme Übelohe au lieu de capituler. Car l'existence est une lutte pour la dignité - non plus une quête du bonheur comme chez Wedekind.

13 Le texte de Dürrenmatt entretient des relations privilégiées avec celui de Wedekind. Le dramaturge suisse a entrepris de dialoguer avec son confrère allemand, non par la simple juxtaposition mais par le croisement des thèmes, des motifs et par la composition. Dürrenmatt a "réécrit" Wedekind. Il n'aurait pas réécrit Brecht par exemple, dont il a étudié et commenté la dramaturgie ${ }^{34}$. Les quelques exemples de réécriture que nous avons essayé de mettre en lumière témoignent en revanche de la productivité du texte de Wedekind. Dürrenmatt a fait émerger la structure profonde de Der Marquis von Keith et quelques unes des intentions contenues dans ce drame, même si elles apparaissent plutôt en filigrane dans Die Ehe des Herrn Mississippi. Dans son introduction au volume intitulé Réécritures: Heine, Kafka, Celan, Müller, Christian Klein souligne que "la manipulation créatrice dudit texte n'annule, ni n'efface rien, elle instaure un dialogue $\aleph^{35}$. Dürrenmatt a procédé à une réception productive du texte de Wedekind Der Marquis von Keith par le biais d'actualisations et de réappropriations mais aussi de nombreuses créations personnelles. Die Ehe des Herrn Mississippi - comme beaucoup d'autres œuvres de Dürrenmatt - dévoile la fécondité de cette technique d'écriture. Ceci ne doit cependant pas faire oublier que la spécificité du modèle est déterminante : c'est la richesse du drame de Wedekind et de son œuvre en général qui lui a valu l'intérêt que Dürrenmatt a manifesté pour son théâtre.

\section{NOTES}

1. Le journal Abendzeitung reprend les griefs formulés par Tilly Wedekind dans son édition du 2 juin 1952: «Dort heiratet die Witwe den Mann, der ihren Gatten im Duell getötet hat, hier heiratet die Mörderin ihres Gatten den Mann, der seine Frau ermordete, die mit dem Ermordeten ein Verhältnis hatte. So sind natürlich auch alle wesentlichen Übergangsrepliken von auffallender Ähnlichkeit ». In Abendzeitung, München 2. 6. 1952.

2. «(...) auf den Marquis von Keith, ein Theaterstück, das ich für Wedekinds bestes halte und welches mich auf die Idee brachte, die Menschen als Motive einzusetzen. In diesem Stück ging mir die Möglichkeit einer Dialektik m i t Personen auf. (...) Auch dies ist natürlich nicht neu (...) Doch bei Wedekinds Marquis von Keith zeigte sich dieser Kunstgriff eben $\mathrm{m}$ i $\mathrm{r}$ besonders deutlich, und damit hatte ich ein Prinzip gefunden, induktiv zu schreiben und meine fünf 
Hauptpersonen zu finden, indem ich eine aus der andern entwickelte und so fort. », Friedrich Dürrenmatt, in Die Tat, 9 août 1952, Zurich.

3. «So wichtig auch Wedekind sein mag, die Möglichkeit in Wedekind ist wichtiger : Es ist eine Möglichkeit der Komödie. H i e $r$ habe ich eingesetzt. Ich glaube nicht, daß ein heutiger Komödienschreiber an Wedekind vorbeigehen kann, wie mir dies Frau Tilly offenbar zumutet. » ib.

Voir aussi ce que Dürrenmatt écrira au sujet de la comédie en 1954 dans ses Theaterprobleme : «Die Tragödie setzt Schuld, Not, Maß, Übersicht, Verantwortung voraus. In der Wurstelei unseres Jahrhunderts, in diesem Kehraus der weißen Rasse, gibt es keine Schuldigen und auch keine Verantwortlichen mehr. Alle können nichts dafür und haben es nicht gewollt. Es geht wirklich ohne jeden. Alles wird mitgerissen und bleibt in irgendeinem Rechen hängen. Wir sind zu kollektiv schuldig, zu kollektiv gebettet in die Sünden unserer Väter und Vorväter. Wir sind nur noch Kindeskinder. Das ist unser Pech, nicht unsere Schuld gibt es nur noch als persönliche Leistung, als religiöse Tat. Uns kommt nur noch die Komödie bei ». In Friedrich Dürrenmatt, Gesammelte Werke. Essays, Gedichte, Diogenes Taschenbuch 22850, p. 59. La théorie de la comédie que Dürrenmatt énonce ici peut se lire comme une analyse du drame de Wedekind, Der Marquis von Keith.

4. «Der Saatsanwalt Mississippi sei eine Weiterführung der Gestalt des Romulus, der sich ja auch damit rechtfertige, daß er ein Richter sei. Die Gestalt des Mississippi sei nichts anderes als eine Kritik, die der Autor an einer seiner Gestalten ausübe. Aus dem Verhältnis Romulus-Julia, das ebenfalls eine Ehe mit einer bestimmten Absicht sei, habe sich folgerichtig die Ehe MississippiAnastasia entwickelt. Die Dialektik sei dieselbe, wenn auch das Verbrechen nicht dasselbe sei. » : Friedrich Dürrenmatt, ,Bekenntnisse eines Plagiators', in Abendzeitung, München 2.6.1952.

5. Friedrich Dürrenmatt, Die Ehe des Herrn Mississippi. Komödie und Drehbuch, Werkausgabe in siebenunddreißig Bänden, vol. 3, Diogenes, p. 117.

6. Dürrenmatt accompagne la version de 1980 de l'avertissement suivant: «(...) bildet die vorliegende Fassung eine Art Synthese : Es galt, Kühnheiten wiederaufzunehmen, die ich nur in der ersten Fassung wagte, und Erfahrungen beizubehalten, die nach und nach kamen. Im großen und ganzen ist der Erste Teil der zweiten und der Zweite Teil der dritten Fassung nachgebildet (...) ».

7. Voir Peter André Bloch, ,Tentative de représentation de l'irreprésentable. Achterloo de Friedrich Dürrenmatt ou la dissolution du héros dramatico-historique' in Les Cahiers du Littoral, Jacqueline Bel (éd.), I/ n²1, juin 2000, pp. 54-55.

8. Gerwin Marahrens, ,Frank Wedekinds Der Marquis von Keith und Friedrich Dürrenmatts Die Ehe des Herrn Mississippi', in Momentum dramaticum, 1990, pp. 493-519.

9. Voir l'article de Gerwin Marahrens, pp. 494 et 518. Il cite à ce propos les travaux de Wilfried Berghahn ('Friedrich Dürrenmatts Spiel mit den Ideologien', in Frankfurter Hefte 11 (1956)), Christian Markus Jauslin (,Friedrich Dürrenmatt : Zur Struktur seiner Dramen', in Juris, Zürich, 1964), Hans Jochen Irmer (,Friedrich Dürrenmatt', in Der Theaterdichter Frank Wedekind: Werk und Wirkung, Berlin, DDR, Henschel, 1975), Urs Jenny ('Dürrenmatt : A Study of His Plays', London : Methuen, 1978), Kenneth S. Whitton ('The Theatre of Dürrenmatt : A Study in the Possibility of Freedom', London : Wolff, 1980), Gerhard P. Knapp (Friedrich Dürrenmatt, Stuttgart,

Metzler 1980, pp. 51-52), Jennifer E. Michaels ('Vom Romulus zum Engel', in Zu Friedrich Dürrenmatt, hrsg. Armin Arnold, Klett, 1982) et de Jan Jopling Seiler (Wedekind and Dürrenmatt : A Comparative Study, Dissertation, University of Wisconsin, 1973).

10. Frank Wedekind, Der Marquis von Keith, Goldmann 889, p. 163.

11. Ib., p. 94.

12. Friedrich Dürrenmatt, Die Ehe des Herrn Mississippi, Werkausgabe in siebenunddreißig Bänden, vol. 3, Diogenes, p. 112. 
13. Wedekind a défini le drame Der Marquis von Keith comme étant « das Wechselspiel zwischen einem Don Quixote des Lebensgenusses und einem Don Quixote der Moral». Dans la pièce de Wedekind, la figure de Don Quichotte est apparentée à l'insuccès permanent. Chez Dürrenmatt, c'est Graf Bodo von Übelohe-Zabernsee qui revêt les oripeaux de Don Quichotte, résumant ainsi la philosophie de l'existence de l'ensemble des protagonistes : ne jamais capituler, même si le succès de l'entreprise n'est pas immédiat. Dürrenmatt déplace l'accent.

14. «Hier muß eine Bemerkung über Die Ehe des Herrn Mississippi eingeschoben werden. Diese Komödie ist ein künstlerisches Experiment, mehr in ihr zu sehen, etwa gar eine Technik, die ich nun auch des weiteren anzuwenden im Sinne hätte, wäre Unsinn (...) » : Friedrich Dürrenmatt, in Die Tat, 9 août 1952, Zurich.

15. Gerwin Marahrens, ,Frank Wedekinds Der Marquis von Keith und Friedrich Dürrenmatts Die Ehe des Herrn Mississippi', in Momentum dramaticum, 1990, p. 516.

16. Dans un article de Die Zeit du 16 août 1985, Dürrenmatt définit et justifie l'écriture par la pensée: «Für mich ist Schreiben vor allem Denken». Le 10 janvier 1981, à l'occasion du soixantième anniversaire de l'auteur, Hugo Loetscher avait déjà qualifié Dürrenmatt de «Gedankendramaturg »: «Dieser Gedankendramaturg ist nicht vom Theaterautor und vom Erzähler zu trennen ». Hugo Loetscher, "Ein Gedankendramaturg”, in Über Friedrich Dürrenmatt, Daniel Keel (éd.), Diogenes Taschenbuch 20861, pp. 106-120, ici p. 114.

17. «Ein Zimmer, dessen spätbürgerliche Pracht und Herrlichkeit zu beschreiben nicht eben leicht sein wird. Doch da sich die Handlung in ihm abspielen wird, ausschließlich in ihm, ja, da gesagt werden darf, daß die folgenden Geschehnisse die Geschichte dieses Zimmers darstellen, soll seine Beschreibung gewagt sein (...) », in F. D., op. cit., p. 11.

18. «In der Mitte ein rundes Biedermeier-Kaffeetischchen, die eigentliche Hauptperson des Stücks, um das herum sich das Spiel dreht (...) » ib., p. 12.

19. «Der Ort ist derselbe, doch leider, leider stark verändert : Die Tapete dahin, die Liebesgöttin kaum noch zu erraten, die Louis-Quatorze-, Quinze-, Seize-Möbel demoliert, und nur noch der Biedermeier-Kaffeetisch ist zum Glück heil geblieben. » id., p. 102.

20. Le rideau se ferme, la lumière s'allume et Übelohe apparaît sur l'avant-scène pour indiquer au public que ce n'est pas encore l'entracte. Il commente alors la manière dont Dürrenmatt a imaginé cette pièce, renseigne le public sur le passé de Mississippi et de Saint-Claude et anticipe la fin de la pièce. Enfin, il situe son propre personnage dans le déroulement des événements. Voir la harangue de Übelohe, pp. 56-60 ; Saint-Claude se prête également à cet exercice au tout début de la pièce, après avoir été abattu par trois hommes vêtus d'imperméables. Son monologue équivaut à l'exposition. Voir le discours de Saint-Claude à l'adresse du public, pp.13-17; Ce rôle de récitant est également dévolu à Mississippi qui fait le point sur son union avec Anastasia et sur l'idéologie dont il est le représentant. Voir le plaidoyer de Mississippi, pp. 38-41.

21. Dans le monologue de 'Der Minister Diego' s'entrelacent un résumé de la situation, ses commentaires à propos de celle-ci et des indications scéniques. Voir le monologue de ce personnage pp.78-79. Dürrenmatt emploie aussi ce procédé lors des interventions de SaintClaude, Übelohe et Mississippi auxquelles nous faisons allusion dans la note précédente.

22. Übelohe préfère boire du Cognac au lieu du café qu'on lui a servi après avoir appris que Mississippi a aussi empoisonné sa femme avec le poison que lui-même avait fourni à Anastasia : « Du kannst doch unmöglich von mir verlangen, daß ich in diesem Hause noch Kaffee trinke. », in F. D., op. cit., p. 69. Anastasia veut empoisonner Saint-Claude parce que celui-ci veut l'obliger à fuir avec elle au Portugal. Mais Saint-Claude s'en doute, ne boit pas son café mais celui d'Anastasia une fois qu'elle a eu quitté la pièce (pp.99-100). Après le départ de Saint-Claude, Mississippi pénètre dans la pièce, remplit la tasse d'Anastasia de café et y met un morceau de sucre empoisonné (p. 101). Anastasia arrive sur ces entrefaites, tous deux prennent place autour de la petite table ronde et boivent leur café sans savoir que chacun a empoisonné le café de l'autre (p. 103). 
23. « (...) in den Tiegel seiner Komödie zu werfen (...) », in, F. D., op. cit., p. 58.

24. «Es gibt keine Ideen, seien sie sozialer, wissenschaftlicher oder künstlerischer Art, die irgend etwas anderes als Hab und Gut zum Gegenstand hätten. (...) Und glauben Sie ja nicht, daß sich die Welt hierin jemals ändert. » ib., p. 84.

25. « Er ist nämlich wirklich Moralist, von Natur und aus Überzeugung. » id., p. 115.

26. « (...) die Welt von Grund auf durch das Gesetz Mosis zu restaurieren (...) » id., p. 39.

27. «Meine Macht ist nicht zu treffen. Sie gründet sich nicht auf die Leidenschaft der Menschen, sie gründet sich auf ihre Müdigkeit. Die Sehnsucht nach Veränderung ist groß, doch die Sehnsucht nach Ordnung ist stets noch größer. Sie wird mich an die Macht bringen. » id., p. 63.

28. «(...) denn es geht um das nicht unbedenkliche Schicksal dreier Männer (...) die sich aus verschiedenen Motiven nichts mehr und nichts weniger in den Kopf gesetzt hatten, als die Welt teils zu ändern, teils zu retten, und denen nun freilich das grausame Pech zustieß, mit einer Frau zusammenzukommen (...) die weder zu ändern, noch zu retten war, weil sie nichts als den Augenblick liebte (...)» id., p. 15.

29. «Während ich, Macht begehrend und nichts anderes, die Welt umarme - Anastasia hat sich erhoben und geht zum Minister, der sie umfängt. » id., p. 112.

30. Friedrich Dürrenmatt expose ses idées sur la tâche dévolue au théâtre dans un discours de 1959 : « Nicht, daß die Schriftsteller anderer Epochen naive Naturburschen gewesen wären, aber ihr Bewußtsein lag in der Form, ihre Stile bewältigten alles, ihre Rethorik verarbeitete jede Fabel. Wir sind auch bewußter dem Inhalt gegenüber geworden (...) : muß (ein Theaterstück) eine Aussage besitzen oder nicht? (...) Unter einer Aussage wollen wir hier etwas Allgemeingültiges verstehen. Der Sinn der Behauptung, daß ein Theaterstück eine Aussage besitzen soll, liegt darin, daß aus etwas Speziellem, Besonderem, das ja jede Handlung eines Theaterstücks darstellt, etwas Allgemeines herausspringen soll, eine allgemeine Erkenntnis, ein Resultat.» Friedrich Dürrenmatt, 'Vom Schreiben', in F. D., Literatur und Kunst. Essays und Reden, Diogenes 20857, pp. 78-79.

31. «(..) sondern daß es ihm darum ging, zu untersuchen, was sich beim Zusammenprall bestimmter Ideen mit Menschen ereignet, die diese Ideen wirklich ernst nehmen und mit kühner Energie, mit rasender Tollheit und mit einer unerschöpflichen Gier nach Vollkommenheit zu verwirklichen trachten (...) ", in F. D., op. cit., p. 57.

32. Les indications scéniques associent l'identité de Don Quichotte de la Mancha à Übelohe : «Im Fenster links erscheint Übelohe, allein sichtbar, einen verbeulten Helm aus Blech auf dem Kopf, eine verbogene Lanze in der Rechten, immer wieder getaucht in den kreisenden Schatten einer Windmühle. » ib., p. 113.

33. «Mein Auftritt ist lächerlich, mehr als lächerlich, unzeitgemäß, wie ich selbst, wie mein groteskes Leben. »id., p. 57.

34. Dans ses Theaterprobleme, Dürrenmatt porte un jugement sur la conception théâtrale de Bertolt Brecht qu'il exprime en ces termes : «Die Brechtsche These, die er in seiner Straßenszene entwickelt, die Welt als Unfall hinzustellen und nun $\mathrm{zu}$ zeigen, wie es $\mathrm{zu}$ diesem Unfall gekommen sei, mag großartiges Theater geben, was ja Brecht bewiesen hat, doch muß das meiste bei der Beweisführung unterschlagen werden: Brecht denkt unerbittlich, weil er an vieles unerbittlich nicht denkt ", in Friedrich Dürrenmatt, op. cit., p. 64.

35. Réécritures: Heine, Kafka, Celan, Müller. Essais sur l'intertextualité dans la littérature allemande au $\mathrm{XX}^{e}$ siècle, Christian Klein (éd.). Presses Universitaires de Grenoble. Ludwigsburger Hochschulschriften 11, 1989. 


\section{RÉSUMÉS}

La première de Die Ehe des Herrn Mississippi a eu lieu aux « Münchner Kammerspiele » le 26 mars 1952. Quelques mois plus tard, Tilly Wedekind, la veuve de Frank Wedekind, porte plainte auprès $\mathrm{du}$ «Schutzverband deutscher Schriftsteller» contre Friedrich Dürrenmatt pour avoir plagié dans Die Ehe des Herrn Mississippi une pièce de Wedekind: Schloß Wetterstein. Dürrenmatt prend position contre les accusations de Tilly Wedekind dans 'Bekenntnisse eines Plagiators', article publié dans Die Tat du 9 août 1952. Dans ces 'Bekenntnisse' il dit regretter que Tilly Wedekind n'ait pas remarqué que la seule œuvre à avoir véritablement influencé Die Ehe des Herrn Mississippi était Der Marquis von Keith. C'est à la lecture de cette œuvre qu'il a vraiment compris l'importance de concevoir les personnages de manière dialectique et qu'il a élaboré les principes de leur utilisation comme types et celui de l'écriture inductive. En outre, Der Marquis von Keith lui a donné l'idée d'écrire des comédies.

L'accusation de plagiat de Tilly Wedekind se borne à relever quelques chutes. Mais le résultat obtenu par Dürrenmatt montre qu'il a osé suivre son inspiration et qu'il a su trouver ses propres sujets. Dürrenmatt considérait cette comédie comme une œuvre expérimentale. Il est manifeste que la motivation des deux auteurs était différente. L'analyse des deux pièces de théâtre montre l'influence de Wedekind sur le dramaturge suisse et le champ d'expérimentation que Die Ehe des Herrn Mississippi constituait effectivement pour Dürrenmatt, qui ne montre pas la réalité mais une réalité. Il ne lui importe pas seulement que son théâtre reflète quelque chose, la manière dont il reflète les choses est tout aussi importante.

Am 26. März 1952 wurde Die Ehe des Herrn Mississippi in den Münchner Kammerspielen uraufgeführt. Einige Monate später erhob Tilly Wedekind, die Witwe Frank Wedekinds, vor dem Schutzverband deutscher Schriftsteller gegen Friedrich Dürrenmatt die Anklage, mit der Komödie Die Ehe des Herrn Mississippi Wedekinds Werk Schloß Wetterstein abgeschrieben zu haben. Dürrenmatt nimmt Stellung zu Tilly Wedekinds Anschuldigungen in 'Bekenntnisse eines Plagiators' in Die Tat vom 9. August 1952. In diesen 'Bekenntnissen' bedauert er, daß Tilly Wedekind nicht festgestellt habe, Wedekinds Theaterstück Der Marquis von Keith sei das einzige Werk, welches wirklich auf Die Ehe des Herrn Mississippi eingewirkt habe. Dabei sei ihm nämlich die Dialektik mit Personen aufgegangen, woraus er das Prinzip entwickelte, die Menschen als Motive einzusetzen und induktiv zu schreiben. Ferner brachte ihn Wedekinds Der Marquis von Keith auf die Idee der Komödie.

Tilly Wedekinds Anklage hängt an einzelnen Pointen. Das Resultat von Dürrenmatts Schreiben zeigt aber, daß er es wagte, sich seinen Einfällen hinzugeben und der Anforderung gerecht zu werden, eigenen Stoff zu finden. Dürrenmatt verstand diese Komödie als ein künstlerisches Experiment. In der Tat sind Dürrenmatts und Wedekinds Motive völlig verschieden. Die Untersuchung beider Theaterstücke ergibt, daß Wedekind auf den Schweizer Dramatiker zwar einwirkte, Dürrenmatt aber in Die Ehe des Herrn Mississippi experimentierte, nicht die Wirklichkeit, sondern eine Wirklichkeit zeigte. Ihm kommt es nicht nur auf das an, was er widerspiegelt, sondern auch darauf wie er die Dinge widerspiegelt.

\section{AUTEUR}

\section{JACQUELINE BEL}

Université du Littoral-Côte d'Opale, Boulogne-sur-Mer 